\title{
LIGNIN BIO-BASED MATERIAL IN UNSATURATED POLYESTER
}

\author{
TRI-DUNG NGO,,$^{*, * *, * * *}$ ERIC PATENAUDE ${ }^{* * *}$ and BEHZAD AHVAZI ${ }^{*, * *, * * *}$ \\ *InnoTech Alberta Inc., 250 Karl Clark Road, Edmonton, Alberta, T6N 1E4, Canada \\ ** University of Alberta, Faculty of Engineering, Donadeo Innovation Centre for Engineering, \\ 9211-116 Street NW, Edmonton, Alberta, T6G 1H9, Canada \\ ***N National Research Council Canada, 75 De Mortagne Blvd., \\ Boucherville, Quebec, J4B 6Y4, Canada \\ $₫$ Corresponding author: T.-D. Ngo, tridung.ngo@innotechalberta.ca
}

Received November 16, 2018

The potential utilization of lignin, as a natural-based material, in unsaturated polyester (UPE) has been investigated. Kraft (Indulin AT) and sulfite lignin (ArboSO1) samples from forestry biomass and their consistencies in UPE were evaluated by qualitative and quantitative methods. The tensile and impact properties of the cured UPE with and without lignins, as well as fracture surfaces, were also studied. The result indicated that the ash content is quite high for lignosulfonate ArboSO1. NMR spectra show significant differences almost in the entire spectral region for the two lignins. The aliphatic hydroxyl groups were found more uniform in Indulin AT than in ArboSO1. Indulin AT seems to have more pronounced condensed units than ArboSO1. The guaiacyl units in Indulin AT were found to be more uniform and of higher magnitude than in ArboSO1, similarly to free phenolic units. However, the terminal carboxylic content in ArboSO1 was found to be higher than in Indulin AT. The investigation by SEM showed that the particles of the two lignins are porous and have different sizes, mostly less than $100 \mu \mathrm{m}$. The addition of different concentrations of lignins increased the viscosity of UPE; however, the viscosity was still suitable for processing at the lignin loading levels. The results also indicated that the lignin types and loadings did influence the tensile properties, but not the impact property of the UPE. The modulus of UPE decreased almost linearly with the loading of ArboSO1 for both UPEs. On the other hand, the modulus of UPE leveled up for Indulin AT only at 10 and $20 \mathrm{wt} \%$ loading. The positive effect of adding $10 \mathrm{wt} \%$ of both lignin samples on the tensile strength of UPE was demonstrated. The presence of lignin affected the fracture surface of the UPE systems, regardless of the lignin type and consistency. This novel approach could reduce costs and environmental impact of manufacturing UPE products, while improving their quality.

Keywords: lignin, unsaturated polyester, biomaterial, biocomposite

\section{INTRODUCTION}

Polymer composites have received growing attention in several industries, such as plastics, automotive, aviation, textiles, etc. In the past decades, inorganic fillers have been utilized exclusively in polymer composites manufacturing. A commercial example of commonly used thermosetting matrix is unsaturated polyester (UPE). UPE has various properties, among other types of thermosets, such as its relative affordability, moldability at room temperature, and resistance to thermal and weather conditions. ${ }^{1,2}$

With ever-increasing global concern over climate changes, irreversible environmental damages, and rapid depletion of non-renewable resources, there is a strong worldwide interest in developing suitable technologies that can derive chemicals and materials from renewable biomass, such as lignin in polymer composites formulation. Lignin is a class of complex organic polymers that form key structural materials in the support tissues of vascular plants and some algae. ${ }^{3}$ Lignins are particularly important in the formation of cell walls, especially in wood and bark, because they lend rigidity and resistance to rotting. Chemically, lignins are considered as cross-linked phenolic polymers. ${ }^{4}$ Lignin is the second most naturally abundant bio-polymeric substance after cellulose; the natural polymer makes up to $40 \%$ of forestry and up to $30 \%$ of certain ligno-cellulosic materials in agricultural residues. ${ }^{5,6}$ Several methods, such as kraft process, alkaline treatment, steam explosion and organosolv processes, are utilized for obtaining 
lignin. Although lignin is an attractive abundant and relatively inexpensive biomass resource, due to its complex nature and undefined chemical structure, the industrial applications of lignin are rather limited. However, based on its interesting functionalities and properties, lignin offers perspectives for higher added value applications in renewable products. ${ }^{7,8}$

Presently, lignin is utilized almost exclusively as fuel to power the evaporators of the chemical recovery processes and the liquor concentration system of pulp mills. ${ }^{9,10}$ In recent years, much attention has been devoted to the possibility of replacing petrochemical-based polymer, filler and reinforcement materials by bio-based materials. Due to the competitive properties offered by biopolymers used as matrix, filler or reinforcement, these materials have been considered as the most promising substitute for petrochemical-based polymers. ${ }^{11-13}$ In addition, lignin used as matrix, filler or reinforcement may provide special functional properties, such as oxidation resistance, stabilization, biodegradability, anti-fungal and antibiotic activity and UV-absorption resistance, to polymer composites. $^{14}$

Research interest has been focused on utilizing lignin as bio-polymer or bio-filler ${ }^{15-17}$ in the formulation and preparation of biomaterials. Restrictively, lignin has been used as filler material in thermoplastic polymers, ${ }^{18,19}$ thermosetting polymers ${ }^{20,21}$ and rubbers ${ }^{22,23}$ with limited positive or even negative influences on the material's mechanical properties. In general, the properties of lignin-based composites are far from satisfactory by adding lignin as particulate filler to a polymer. This is mainly because of the complex and heterogeneous structure and chemistry of lignin, which is highly dependent on the lignin sources, recovery and processing conditions. In addition, not many studies have been done on the effect of lignin on the properties of UPE. Therefore, the aim of this investigation was to evaluate the potential of lignin as a natural material in unsaturated polyester, with a view to substituting as much as possible the unsaturated polyester by the lignin, in order to increase the green portion and reduce the production cost, with minor impact on the composite performance. In addition, the selection of the right lignin for the polymers is also a challenge and is not well understood. In this study, two types of lignin from different processes, namely kraft (Indulin AT) and sulfite (ArboSO1) lignins, were selected and characterized by qualitative and quantitative methods. The characterization and selection of lignins as raw materials would then pave the way to prepare a bio-based UPE with similar characteristics. The influences of the lignin types and their loading on the properties of two different unsaturated polyesters were also investigated. The utilization of the lignin polymer with highly branched polyphenolic macromolecules as a raw material in substituting UPE would then be a new step towards preparing bio-based UPE materials for industrial applications. The use of green, natural-based materials in manufacturing engineered products would further enhance their green credentials.

\section{EXPERIMENTAL \\ Materials}

Unsaturated polyester resins R937-EPE-50 and RL2710 (so-called R9 and R2, respectively) were obtained from AOC and CCP composites (Progress), respectively. Luperox DDM-9 (mixture of methyl ethyl ketone peroxide - MEKP) from Arkema Inc., was used as an initiator at $1.5 \mathrm{wt} \%$ of the resin. Softwood kraft lignin (Indulin AT) and sodium lignosulfonate (ArboSO1) from MeadWestwaco and Tembec (Temiscaming, Québec, Canada) were used in this study. All the chemicals and reagents utilized for the NMR study were purchased from Sigma-Aldrich Chemicals.

\section{Preparation of lignin UPE}

Lignin samples were dried at $60{ }^{\circ} \mathrm{C}$ under vacuum for at least 4 hours prior to their utilization. Thereafter, lignins were pre-mixed with UPE using a conventional mechanical stirrer. The desired amounts of Indulin AT and ArboSO1 lignins were added slowly to R937-EPE50 and RL2710 UPE resins, as specified in Table 1. Lignin concentrations in the resin system were varied from 10 to $40 \mathrm{wt} \%$. The resulting mixtures were stirred at room temperature for $10 \mathrm{~min}$ at $1200 \mathrm{rpm}$.

For curing the UPE and UPE-lignin resins, Luperox DDM-9 at $1.5 \mathrm{wt} \%$ of the resins was added to the UPE and UPE-lignin resins. The resulting mixtures were mixed by hand for $2 \mathrm{~min}$ and subjected to degassing under vacuum at room temperature for $5 \mathrm{~min}$. The UPE derived samples with and without lignins were then casted in aluminum molds. The casted resins were cured at room temperature for 1 day and subsequently post-cured at $100{ }^{\circ} \mathrm{C}$ for $1 \mathrm{~h}$. After curing, the samples were demolded and cut to dog-bone and rectangular shaped specimens, according to the corresponding ASTM standards for tensile and impact tests, respectively. 
Table 1

Formulation of UPE-lignin

\begin{tabular}{ccccc}
\hline Sample & $\mathrm{R} 2(\mathrm{wt} \%)$ & $\mathrm{R} 9(\mathrm{wt} \%)$ & $\mathrm{Ab}(\mathrm{wt} \%)$ & $\mathrm{AT}(\mathrm{wt} \%)$ \\
\hline $\mathrm{R} 2$ & 100 & & & \\
$\mathrm{R} 2-10 \mathrm{Ab}$ & 90 & & 10 & \\
$\mathrm{R} 2-20 \mathrm{Ab}$ & 80 & & 20 & \\
$\mathrm{R} 2-30 \mathrm{Ab}$ & 70 & & 30 & \\
R2-40Ab & 60 & & 40 & 10 \\
R2-10AT & 90 & & & 20 \\
R2-20AT & 80 & & & 30 \\
R2-30AT & 70 & & & \\
\hline R9 & & 100 & 10 & \\
R9-10Ab & & 90 & 20 & \\
R9-20Ab & & 80 & 30 & \\
R9-30Ab & & 70 & 40 & 10 \\
R9-40Ab & & 60 & & 20 \\
R9-10AT & & 90 & & 30 \\
R9-20AT & & 80 & & \\
R9-30AT & & 70 & & \\
\hline
\end{tabular}

\section{Characterization \\ Moisture and ash contents}

Lignin samples (3 x $0.5 \mathrm{~g})$ were quantitatively measured in a pre-weighed crucible and placed in an oven at $105 \pm 5{ }^{\circ} \mathrm{C}$ overnight. The samples were then transferred to a desiccator at room temperature over drierite to a constant weight for moisture content determination. After measuring each sample, they were placed in an incinerator at $600{ }^{\circ} \mathrm{C}$ for a period of $3.5 \mathrm{~h}$. The crucibles remained there until the incinerator temperature was $150{ }^{\circ} \mathrm{C}$. Samples were then transferred to a desiccator and allowed to reach a constant weight at room temperature, prior to ash content determination. For each lignin, three replicates of samples were used for determining the moisture and ash contents.

\section{${ }^{31} \mathrm{P}$ nuclear magnetic resonance (NMR) spectroscopy}

Quantitative ${ }^{31} \mathrm{P}$ NMR spectra of all prepared lignins were obtained using published procedures. ${ }^{24-28}$ Approximately 30-40 mg of sample was dissolved in $500 \mu \mathrm{L}$ of anhydrous pyridine and deuterated chloroform $(1.6: 1, \mathrm{v} / \mathrm{v})$ under constant stirring. This was followed by the addition of $100 \mu \mathrm{L}$ of cyclohexanol $(21.38 \mathrm{mg} / \mathrm{mL}$ in pyridine and deuterated chloroform 1.6:1, v/v). This was used as an internal standard and $50 \mu \mathrm{L}$ of chromium (III) acetylacetonate solution $(5.5 \mathrm{mg} / \mathrm{mL}$ in anhydrous pyridine, while for deuterated chloroform 1.6:1, v/v) was used as relaxation reagent. Finally, $100 \mu \mathrm{L}$ of phosphitylating reagent (2-chloro-4,4,5,5-tetramethyl-1,3,2dioxaphospholane, TMDP) was then added and the vial was sealed and shaken to ensure thorough mixing. The mixture was transferred into a $5 \mathrm{~mm}$ NMR tube for subsequent NMR analysis.

All NMR experiments were carried out at $298 \mathrm{~K}$ on a Varian Mercury $400 \mathrm{MHz}$ NMR spectrometer, operated with a $5 \mathrm{~mm}$ broadband inverse probe. ${ }^{31} \mathrm{P}$ NMR spectra were recorded with the spectral width of $40650.406 \mathrm{~Hz}$, operated at spectrometer frequency of $161.98 \mathrm{MHz}$. A relaxation delay of $5 \mathrm{~s}$ was used for 512 scans. The ${ }^{31} \mathrm{P}$ chemical shifts were referenced against a water signal at $132.2 \mathrm{ppm}$. All chemical shifts are reported relative to the product of TMDP with cyclohexanol, which has been observed to give a sharp signal at $145.15 \mathrm{ppm}$ referenced from the water signal (132.2 ppm) for a phosphitylating reagent. The content of hydroxyl groups was obtained by integrating the following spectral regions: aliphatic hydroxyls (150.4$145.5 \mathrm{ppm}$ ); condensed phenolic units (DPM: 144.4143.1; 4-O-5': 143.1-141.7 and 5-5': 141.7-140.8 ppm); syringyl phenolic units (143.1-141.7 ppm); guaiacyl phenolic hydroxyls (140.3-138.3 ppm); $p$ hydroxyphenyl phenolics (138.3-137.3 ppm and 136.8$136.5 \mathrm{ppm})$; and carboxylic acids (135.9-134.0 ppm).

\section{Gel permeation chromatography (GPC)}

Gel permeation chromatography (GPC) analysis was performed using a multi-detection system from Viscotek (Houston, TX), consisting of a Model 302 multiple detector platform, including a PDA detector, refractive index detector, a four capillary viscometer and a 2 angles laser light scattering detector (GPC max integrated pump, auto sampler and degasser). The light scattering detector was not equipped with fluorescence filters and was only used for qualitative monitoring. The software OmniSEC from Viscotek was used for data collection and calibration. Separation was performed in tetrahydrofuran (THF) by injecting 100 $\mu \mathrm{L}$ of $1.5-2 \mathrm{mg} / \mathrm{mL}$ solutions of acetylated lignin in THF into thermostatically controlled SuperRes columns $\left(35{ }^{\circ} \mathrm{C}\right.$; PAS-102, PAS102.5, PAS-103 L, each $300 \mathrm{~mm} \times 8 \mathrm{~mm}$; PolyAnalytik at London, ON). The flow rate was of $1 \mathrm{~mL} / \mathrm{min}$. Molar masses of the 
samples were determined with universal calibration with the refractive index detector and the viscosity detector. The universal calibration curve was based on polystyrene standard from Polymer Laboratories (Amherst, MA) and Sigma-Aldrich (Oakville, ON) using the molar masses determined by the manufacturer and the intrinsic viscosities measured by the apparatus.

\section{Fourier transform infrared (FTIR) spectroscopy}

FT-IR spectra were obtained for powdered solid lignin using a Bruker Tensor Series FT-IR Spectrometer in the Attenuated Total Reflectance (ATR) analysis mode. Spectra were collected from 4000 to $500 \mathrm{~cm}^{-1}$ for 64 scans and $4 \mathrm{~cm}^{-1}$ resolution, using a zinc selenide ( $\mathrm{ZnSe})$ crystal.

\section{Viscosity}

The viscosity of UPE with and without lignin was measured at room temperature on a Brookfield CAP $2000+\mathrm{L}$ viscometer using cone and plate geometry. A small amount of UPE or UPE with lignin samples (less than $1 \mathrm{~mL}$ ) was utilized. The temperature was stabilized to room temperature before running the test.

\section{Morphology}

A JEOL JSM-6100 scanning electronic microscope (SEM) at a voltage of $5 \mathrm{kV}$ was used to observe the lignin's surface and the fracture surface of the cured UPE and UPE-lignin after impact tests. Prior to SEM analysis, a 10-20 nm thin layer of $\mathrm{Pd} / \mathrm{Au}$ was deposited on the sample using a Gatan 682 Precision Etching and Coating System (PECS) in order to minimize the charge effect due to the insulating properties of the samples.

\section{Mechanical properties}

The tensile properties of the unsaturated polyester systems with and without lignins were determined at room temperature and $50 \%$ relative humidity according to ASTM D638-2002 on an Instron 5500R machine, with crosshead speeds of $5 \mathrm{~mm} / \mathrm{min}$. Five replicate of specimens for each unsaturated polyester system with and without lignins were used for the tensile test. The notched Izod impact testing was carried on a TMI tester also at room temperature and $50 \%$ relative humidity according to ASTM D2562002. Ten replicates of specimens for each sample were evaluated for the impact property.

\section{RESULTS AND DISCUSSION}

Indulin AT and ArboSO1 were recovered from kraft and sulfite pulping processes, respectively. Kraft or sulfate pulping processes account for more than $90 \%$ of the chemical pulping process worldwide. Delignification during kraft pulping proceeds in three distinct phases (initial, bulk and final) in an aqueous solution of sodium hydroxide and sodium sulfide. ${ }^{29}$ Lignin is recovered from this process through precipitation by lowering the $\mathrm{pH}$ of the black liquor with either sulfuric acid or carbon dioxide. ${ }^{30}$ The isolated kraft lignin is hydrophobic and contains 1-2\% sulfur by weight as an aliphatic thiol group.

The sulfite process is the second largest chemical pulping process operating on the opposite end of the $\mathrm{pH}$ scale of the kraft process. The lignin isolated from the acid sulfite process is called lignin sulfonate or lignosulfonate. The physicochemical properties of lignosulfonates are affected by the presence of a cationic base, such as calcium, magnesium, sodium and ammonium. ${ }^{31}$ Lignosulfonates are typically highly cross-linked and contain about 5\% sulfur by weight as sulfonate groups. Due to the low $\mathrm{p} K_{\mathrm{a}}$ of sulfonates $\left(\mathrm{p} K_{\mathrm{a}} \leq 2\right)$, lignosulfonates are water soluble lignins.

The lignins isolated from these processes contain several important functional groups, including phenolic hydroxyl, carboxylic groups, and hydroxyl groups, which make lignin a versatile precursor in many formulations, with an overall reactivity that depends entirely on their chemical compositions. ${ }^{32}$ The chemical structure of the lignin polymer consists primarily of phenyl propanoid units, mainly $p$-coumaryl alcohol $(\mathrm{H})$, coniferyl alcohol (G) and/or sinapyl alcohol (S)..$^{33,34}$ These components are cross-linked together in three dimensions via a radical coupling process during biosynthesis. The physical and chemical properties of lignin are highly dependent on the wood species, botanic region, and the isolation processes. ${ }^{35,36}$

The moisture and ash contents for both lignin samples were determined experimentally and are reported in Table 2. The ash content is quite high for lignosulfonate ArboSO1, due to the presence of cationic base during the process.

Quantitative ${ }^{31} \mathrm{P}$ NMR analysis allowed quantifying different classes of $-\mathrm{OH}$ groups of lignin. The technique has been successfully implemented for the absolute determination of various phenolic and non-phenolic structures in several native and technical lignins. ${ }^{37-39}$ The availability of a phosphitylating reagent ${ }^{15}$ that permits excellent resolution and the quantitative spectroscopic detection of several phenolic moieties with varying substitution patterns provide an excellent opportunity for systematically exploring the complex reactions occurring in lignin during several different pulping processes. 
Figure 1 shows the ${ }^{31} \mathrm{P}$ NMR spectra of ArboSO1 and Indulin AT with TMDP. The reagent allows distinguishing several different phenolic condensed units in the lignins, while being less specific for the aliphatic $\mathrm{OH}$ groups. The quantification of all hydroxyl groups, as reported in Table 3, was obtained from the integration of ${ }^{31} \mathrm{P}$ NMR spectra.

Table 2

Moisture and ash contents of lignins

\begin{tabular}{lcc}
\hline Lignin sample & $\begin{array}{c}\text { Average moisture, } \\
(\%)\end{array}$ & $\begin{array}{c}\text { Average ash, } \\
(\%)\end{array}$ \\
\hline Indulin AT & $2.99 \pm 0.20$ & $2.86 \pm 0.24$ \\
ArboSO1 & $0.74 \pm 0.11$ & $24.15 \pm 0.35$ \\
\hline
\end{tabular}

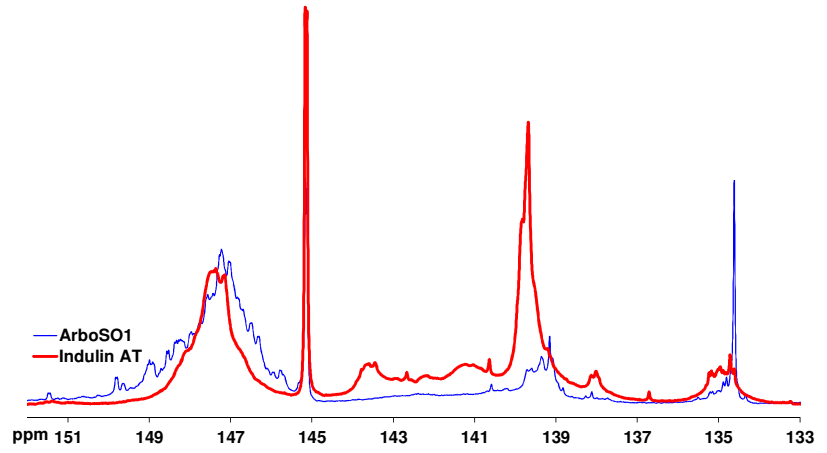

Figure 1: ${ }^{31} \mathrm{P}$ NMR spectra of Indulin AT and ArbroSO1

Another important factor for elucidating the lignin profile is the characterization of its elemental composition. Since the elemental compositions of various types of lignins are exclusively different, the percent elemental composition for each lignin sample, in terms of carbon, hydrogen, nitrogen, oxygen, sulfur, and methoxy content, was analyzed. Based on these data, the empirical formula and the molecular weight of each lignin per C9 unit was calculated and reported in Table 2. Elemental analysis results and the empirical formula of the phenylpropane structural unit of lignin are given in Table 4. In addition, the molecular weights and molecular weight distribution of the lignins determined by GPC are reported in Table 4 .
The comparison of the two NMR spectra shows significant differences almost in the entire spectral region. The aliphatic hydroxyl groups are more uniform in Indulin AT lignin than in ArboSO1. Indulin AT seems to have more pronounced condensed units than ArboSO1. The guaiacyl units in Indulin AT were found to be more uniform and of higher magnitude than those in ArboSO1, similarly to free phenolic units. However, the terminal carboxylic content in ArboSO1 was found to be higher than in Indulin AT. The assignments of the infrared absorption bands in lignin are rather empirical because of the amorphous nature of lignin and the complexity of its chemical components and their random linkages. ${ }^{40}$

Table 3

Quantitative ${ }^{31} \mathrm{P}$ NMR determination of carboxylic, phenolic and primary hydroxyl groups present in lignins

\begin{tabular}{|c|c|c|c|c|c|c|c|}
\hline \multirow{3}{*}{ Lignin sample } & \multicolumn{7}{|c|}{$-\mathrm{OH}(\mathrm{mmol} / \mathrm{g})$} \\
\hline & \multirow{2}{*}{$\mathrm{COOH}$} & \multicolumn{3}{|c|}{ Phenolic } & \multirow{2}{*}{$\begin{array}{c}\text { Condensed } \\
\text { phenolic }\end{array}$} & \multirow{2}{*}{ Primary } & \multirow{2}{*}{$\begin{array}{c}\text { Total } \\
\text { phenolic }\end{array}$} \\
\hline & & $\mathrm{G}^{*}$ & $\mathrm{~S}^{\ddagger}$ & $\mathrm{H}^{\S}$ & & & \\
\hline Indulin AT & 0.21 & 1.80 & 0.00 & 0.12 & 1.28 & 3.27 & 3.20 \\
\hline ArboSO1 & 0.51 & 0.87 & 0.00 & 0.03 & 0.28 & 5.83 & 0.9 \\
\hline
\end{tabular}

*G: guaiacyl phenolic $\mathrm{OH} ;{ }^{\ddagger} \mathrm{S}$ : syringyl phenolic $\mathrm{OH} ;{ }^{\circledR} \mathrm{H}$ : p-hydroxylphenyl phenolic $\mathrm{OH}$ 
Table 4

Empirical formula of phenylpropane structural units of lignins

\begin{tabular}{|c|c|c|c|c|c|c|c|c|}
\hline \multirow{2}{*}{$\begin{array}{l}\text { Lignin } \\
\text { sample }\end{array}$} & \multicolumn{6}{|c|}{ Elemental unit (\%) } & \multirow{2}{*}{ C9 structural unit } & \multirow{2}{*}{$\begin{array}{c}\mathrm{Mw} \\
(\mathrm{g} / \mathrm{mol})\end{array}$} \\
\hline & $\mathrm{C}$ & $\mathrm{H}$ & $\mathrm{N}$ & $\mathrm{O}$ & $\mathrm{S}$ & $\mathrm{OCH}_{3}$ & & \\
\hline Indulin AT & 60.63 & 5.75 & 0.87 & 29.59 & 1.69 & 12.92 & $\begin{array}{c}\mathrm{C}_{9} \mathrm{H}_{8.67} \mathrm{O}_{2.79} \mathrm{~N}_{0.121} \mathrm{~S}_{0.102} \\
\left(\mathrm{OCH}_{3}\right)_{0.809}\end{array}$ & 191.55 \\
\hline ArboSO1 & 40.99 & 4.65 & 37.26 & 1.09 & 1.09 & 6.74 & $\begin{array}{c}\mathrm{C}_{9} \mathrm{H}_{11.25} \mathrm{O}_{5.95} \mathrm{~N}_{0.22} \mathrm{~S}_{0.58} \\
(\mathrm{OCH} 3)_{0.612}\end{array}$ & 255.32 \\
\hline
\end{tabular}

Table 5

General assignments for some lignin functional groups and their IR absorption bands ${ }^{31}$

\begin{tabular}{lc}
\hline Functional groups & IR absorption bands $\left(\mathrm{cm}^{-1}\right)$ \\
\hline OH stretching (H-bonded) & $3600-3100$ \\
OH stretching in methylene and methyl groups & $3100-2800$ \\
$-\mathrm{C}=\mathrm{CH}_{2}$ and $-\mathrm{C}=\mathrm{CH}$ & $3092-2990$ \\
$-\mathrm{CH}_{2}$ and $-\mathrm{CH}$ & 2849 \\
Carboxylic -OH & 2918 \\
Carboxylic acid (high concentration) & $2670-2397$ \\
Unconjugated -C=O stretch & $1742-1710$ \\
$-\mathrm{C}=\mathrm{O}$ & 1771 (aromatic) and 1722 (aliphatic) \\
Aromatic skeletal vibrations & $1601-1501$ and $1425-1430$ \\
$\mathrm{C}-\mathrm{H}$ deformation (asymmetric) & $1460-1470$ \\
C-H deformation (symmetric) & $1370-1365$ \\
Syringyl ring breathing with CO-stretching & $1330-1325$ and $1235-1230$ \\
Guaiacyl ring breathing with CO-stretching & $1275-1270$ \\
Ether C-O & 1213 \\
Aromatic C-H in-plane deformation, guaiacyl-type & $1145-1140$ \\
Aromatic C-H in-plane deformation, syringyl-type & 1130 \\
C-O deformation, secondary alcohol and aliphatic ether & $1085-1090$ \\
Aromatic C-H in-plane deformation, guaiacyl type, and C-O & $1028-1035$ \\
deformation, primary alcohol & 970 \\
=CH out of plane deformation (trans) & $860-750$ \\
Aromatic C-H out of plane (guaiacyl, syringyl, and para- & \\
hydroxyl phenolic) &
\end{tabular}

The general assignments for some lignin functional groups and their IR absorption bands are summarized in Table 5 . $^{41}$

The FTIR spectra for Indulin AT and ArboSO1 lignins are shown in Figure 2 with their corresponding assignments as described below.

Indulin AT: -OH stretch, $3100-3400 \mathrm{~cm}^{-1}$; C-H stretch (methylene and methyl), 2800-3000 $\mathrm{cm}^{-1}$; unconjugated $-\mathrm{C}=\mathrm{O}$ stretch, $1705-1740 \mathrm{~cm}^{-1}$; conjugated $-\mathrm{C}=\mathrm{O}$ stretch, $1675-1655 \mathrm{~cm}^{-1}$; aromatic skeletal vibrations, 1420-1430, 15051515, 1595-1603 $\mathrm{cm}^{-1}$; ether C-O, $1194 \mathrm{~cm}^{-1}$; alcohol C-O, $1040 \mathrm{~cm}^{-1}$ (primary) and $1128 \mathrm{~cm}^{-1}$ (secondary); guaiacyl C-H in plane, $1066 \mathrm{~cm}^{-1}$; guaiacyl C-H out of plane $908-831 \mathrm{~cm}^{-1}$.

ArboSO1: -OH stretch, 3100-3400 cm $\mathrm{cm}^{-1}$ C-H stretch (methylene and methyl), 2800-3000 $\mathrm{cm}^{-1}$; unconjugated $-\mathrm{C}=\mathrm{O}$ stretch, $1705-1740 \mathrm{~cm}^{-1}$; conjugated $-\mathrm{C}=\mathrm{O}$ stretch, $1675-1655 \mathrm{~cm}^{-1}$; aromatic skeletal vibrations, 1420-1430, 15051515, 1595-1603 $\mathrm{cm}^{-1}$; ether C-O, $1194 \mathrm{~cm}^{-1}$; alcohol C-O, $1040 \mathrm{~cm}^{-1}$ (primary) and $1128 \mathrm{~cm}^{-1}$ (secondary); guaiacyl C-H in plane, $1066 \mathrm{~cm}^{-1}$; guaiacyl C-H out of plane $908-831 \mathrm{~cm}^{-1}$.

The FTIR spectra of Indulin AT and ArboSO1 follow a common pattern, with a broad band at $3600-3300 \mathrm{~cm}^{-1}$ corresponding to the hydroxyl groups in phenolic and carboxylic acids and several bands with variable intensity in the fingerprint region (1900 to $\left.800 \mathrm{~cm}^{-1}\right)$. In this region, the $\mathrm{O}-\mathrm{H}$ stretch $\left(3100-3400 \mathrm{~cm}^{-1}\right)$ seems to be less broad for ArboSO1 than for Indulin AT. In addition, the $\mathrm{C}-\mathrm{H}$ stretch in methyl and methylene groups $\left(2800-3000 \mathrm{~cm}^{-1}\right)$ is less available in AbroSOl than in Indulin AT. The next features appear at $1760 \mathrm{~cm}^{-1}$ and are assigned to aromatic acetoxy groups, along with the strong band at $1616 \mathrm{~cm}^{-1}$, due to unconjugated carbonyl- 
carboxyl stretching with two very small shoulders at 1757 and $1706 \mathrm{~cm}^{-1}$ in ArboSO1. The Indulin AT spectrum showed only a small shoulder at 1657 and a strong band at $1601 \mathrm{~cm}^{-1}$. The most significant features in this region were recorded for Indulin AT lignin at $1516 \mathrm{~cm}^{-1}$ due to the $\mathrm{C}=\mathrm{C}$ and aromatic skeletal vibrations, $1462 \mathrm{~cm}^{-1}$ for C$\mathrm{H}$ deformation combined with aromatic ring vibrations. The most noticeable bands for the ArboSO1 sample were recorded at $1224 \mathrm{~cm}^{-1}$ (C$\mathrm{C}, \mathrm{C}-\mathrm{O}$ and $\mathrm{C}=\mathrm{O}$ stretching) and in the region between $654-530 \mathrm{~cm}^{-1}$. The bands for the Indulin AT sample were recorded at $1194 \mathrm{~cm}^{-1}$ (ether C$\mathrm{O}$ ); at $1040 \mathrm{~cm}^{-1}$ (alcohol C-O, primary) and 1128 $\mathrm{cm}^{-1}$ (alcohol C-O, secondary). The band was at $1066 \mathrm{~cm}^{-1}$ for guaiacyl C-H in plane and at 908$831 \mathrm{~cm}^{-1}$ for guaiacyl C-H out of plane.

The bands for the ArboSO1 sample were recorded at $1194 \mathrm{~cm}^{-1}$ (ether $\mathrm{C}-\mathrm{O}$ ); at $1040 \mathrm{~cm}^{-1}$ (alcohol C-O, primary) and $1128 \mathrm{~cm}^{-1}$ (alcohol C$\mathrm{O}$, secondary). The band was at $1066 \mathrm{~cm}^{-1}$ for guaiacyl C-H in plane and at $908-831 \mathrm{~cm}^{-1}$ for guaiacyl C-H out of plane. The band appearing at $617-656 \mathrm{~cm}^{-1}$ is assigned to the sulfonic groups (S-O stretching vibration) formed from the reaction of sodium sulfite with the secondary $\mathrm{OH}$ of the aliphatic side chain of lignins.

The morphology of the lignins was observed under SEM and resulting images are shown in Figure 3. The properties of lignin are related not only to the nature of the hydrophilic groups, which depends upon the pulping process, but also to the size of the polymeric pieces, which in turn depends on the recovery technique used to process the black liquors. The grain diameters and relative proportions of the various size distributions of lignin fragments for differently isolated samples play an important role in prospective biomaterial applications. One can see that the lignin includes round particles, and fragmented and collapsed ones. The two lignin particles are porous and have different sizes, which are mostly less than $100 \mu \mathrm{m}$, although there are a few of them larger than $10 \mu \mathrm{m}$ for Indulin AT. However, the particles seem a bit smaller for ArboSO1 than for Indulin AT. The results also show that more rounded particles were broken down for Indulin AT than for ArboSO1. The overall morphology seems uniform with particles having smooth surfaces. This structure can be influenced by the chemistry, isolation and drying processes of the lignins. The viscosity of UPE resins can impact the processing and subsequently affect the performance of the final products. Therefore, the viscosity of UPE and UPE-lignin is important to be evaluated. The viscosity of the mixtures of two different UPE resins with the two lignins at different lignin loadings was tested. Figure 4 shows the viscosity of UPE resins with and without lignins. In general, the presence of lignin in the two UPE resins changed the rheological behavior of the resins toward non-Newtonian.

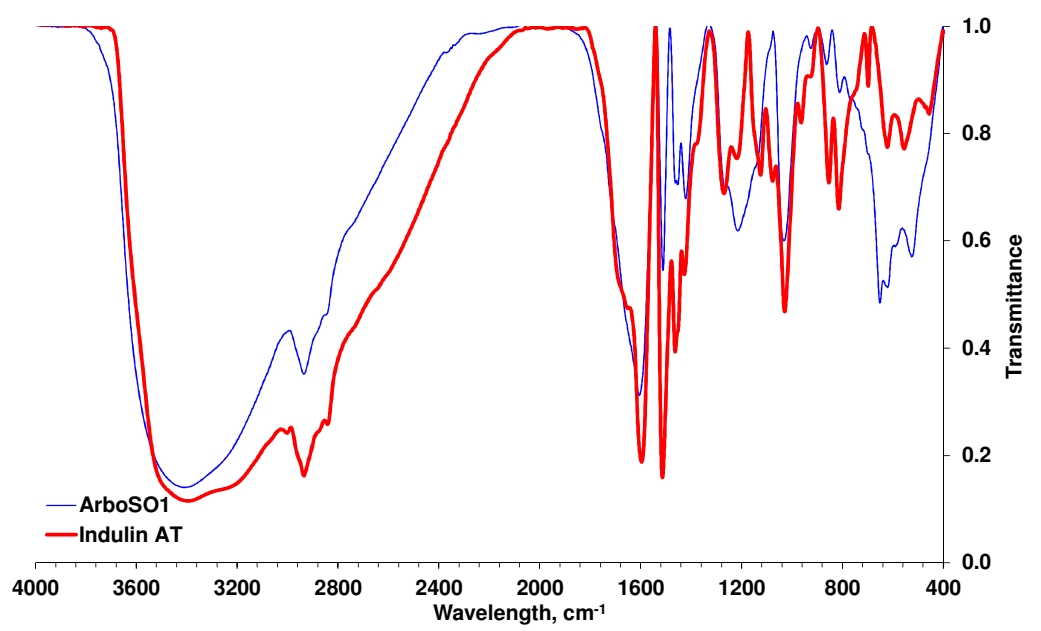

Figure 2: FTIR spectra of Indulin AT and ArboSO1 lignins 

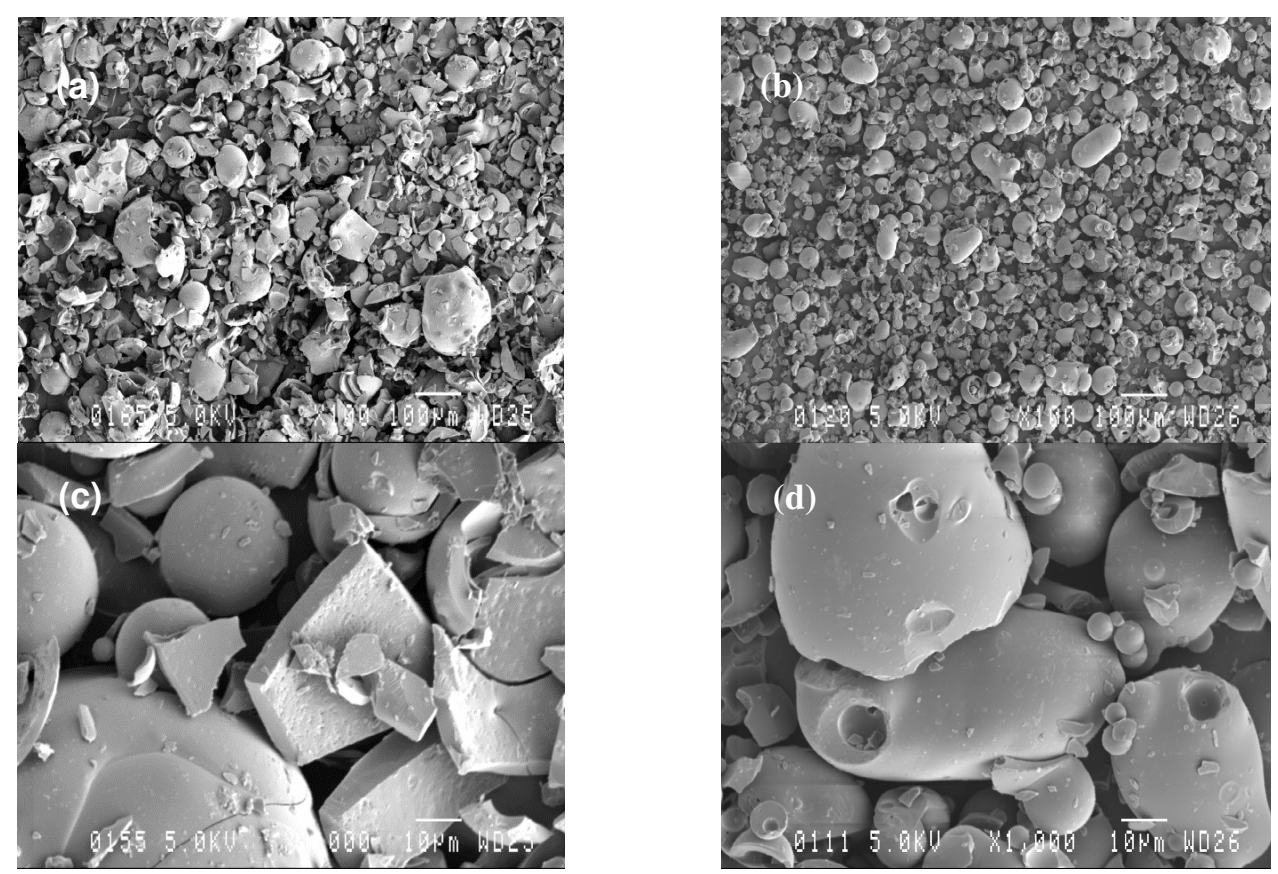

Figure 3: SEM images of Indulin AT (a, c) and ArboSO1 (b, d) under two different magnifications

This means that their viscosities do not remain constant over a given range of shear rates. In the strict sense, the rheological behavior of UPElignin is a combination of non-Newtonian and Newtonian behaviors. At lower shear rates, it behaves as non-Newtonian, but as the shear rate increases, it tends to exhibit a Newtonian behavior. The results show that the presence of lignins increases the viscosity of UPE resins. The results also indicate that the more lignin is added the higher is the viscosity. However, the changes in viscosities were quite complex and closely related to the quality of the lignin dispersion and solubility in the UPE, as well as to the interaction between lignin and UPE molecules. Although the viscosities increased in all the cases, they are still acceptable for processing at loading levels up to $40 \mathrm{wt} \%$ of $\mathrm{Ab}$ and $30 \mathrm{wt} \%$ of Indulin AT in the UPE resins.

The tensile properties of the two UPEs prepared with the two types of lignin at different concentrations are shown in Figure 5. Data indicate the modulus of UPE decreases almost linearly with the ArboSO1 loading for both UPEs. This is similar to the works reported by Yeo et $a l .,{ }^{42}$ where the modulus of the UPE lignin reduced as the lignin loading increased. However, the modulus of the UPE-lignin levels up for Indulin AT only at 10 and $20 \mathrm{wt} \%$ loading. A similar observation was found for both UPE resins. The results also show the positive effect of adding $10 \mathrm{wt} \%$ of both lignin samples on the tensile strength of the UPE. Therefore, lignins are not only considered as filler for UPE, but somehow, they reinforce the UPE resins and improve the tensile strength of the UPE resin. Yeo et $a .^{42}$ also reported increasing numbers of the tensile strength and modulus of UPE-lignin after they modified the lignin with allyltrimethoxy silane. Other works were reported by Ikeda and his team where the tensile stresses of the biocomposites significantly increased with an increase in the lignin content. However, in their study, the matrix was a rubber base. ${ }^{23}$

In our study, the selection of the lignin and its concentration can positively increase the tensile strength without any modifications. However, higher concentrations of both lignins in two different UPE resins have a negative impact on the tensile strength of UPE.

Generally, the mechanical properties of the polymers are influenced by many factors dependent on the structure of polymers, such as primary molecular weight of the polymers, the degree of cross-linking density and regularity of the network structure. ${ }^{43}$ For composite materials, the properties are also affected by the type of reinforcement, the chemistry of the components, 
as well as the interface between the polymers and the reinforcements. In our case, the tensile properties of UPE could have been also influenced by the presence of lignin, lignin type, concentration and the interaction between lignin and UPE. Normally, inert fillers may exhibit very slight changes in the cure properties due to particle surface adsorption effects. However, lignin particles somehow retard the curing of UPE and thus affect their tensile performance.
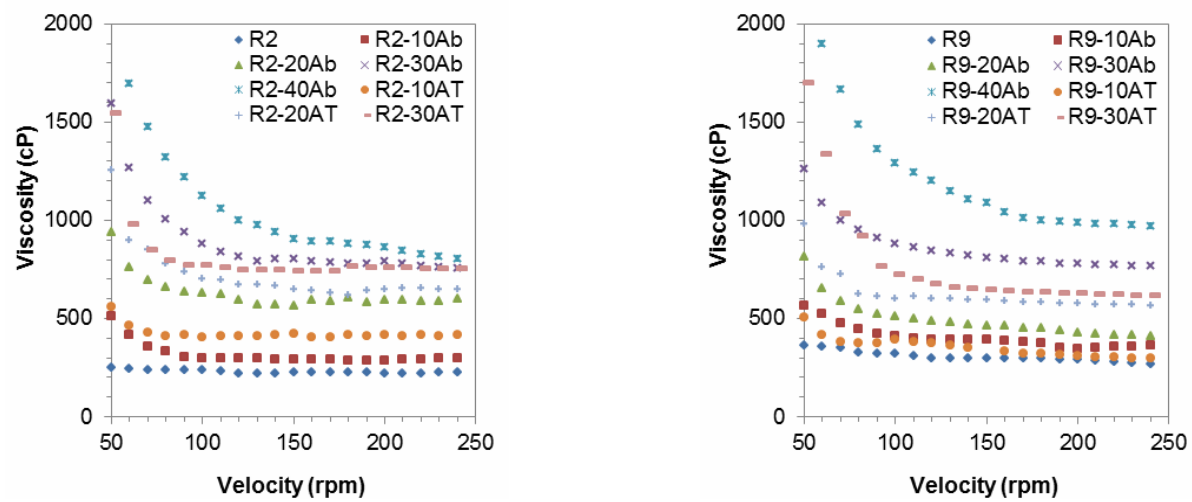

Figure 4: Viscosity of two different UPE resins with Indulin AT and ArboSO1 lignin mixtures at different lignin loadings (left) UPE RL2710 or R2 and (right) R937-EPE-50 or R9
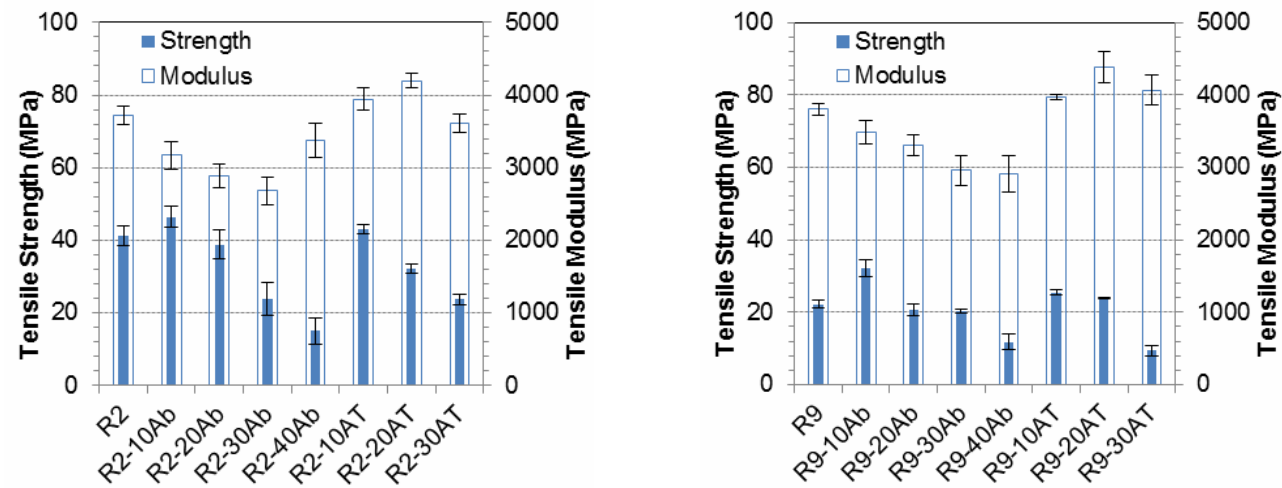

Figure 5: Tensile properties of two different UPE resins with Indulin AT and ArboSO1 (left) UPE RL2710 or R2 and (right) R937-EPE-50 or R9
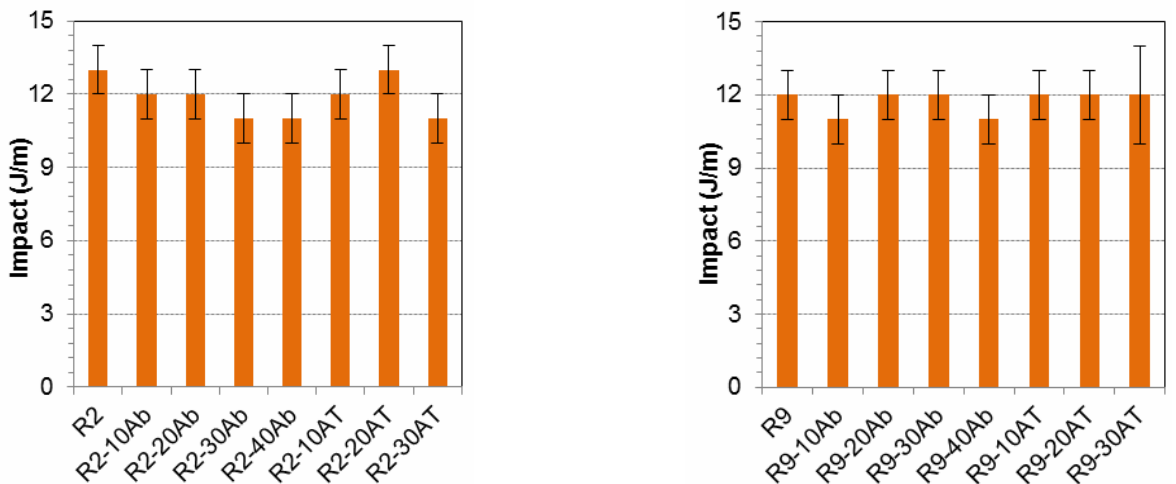

Figure 6: Impact properties of e two different UPE resins with Indulin AT and ArboSO1 (left) UPE RL2710 or R2 and (right) R937-EPE-50 or R9

Impact strength is defined as the resistance of a UPE and UPE-lignin material to impact loading applied in impact testing. Figure 6 shows the impact property of UPE with and without lignins. The results indicate the presence of lignins, their types and contents have no negative effect on the 
impact energy of UPE. The impact property of UPE was greatly reduced with the presence of filler, as compared to the control UPE. Surprisingly, the impact property remains almost the same as for neat UPE for both resins up to 40 wt $\%$ of ArboSO1 and $30 \mathrm{wt} \%$ of Indulin AT. As reported by Yeo et al., the presence of the pristine lignin in the UPE matrix reduced the impact strength of UPE with increased lignin filler loading. ${ }^{42}$

The fracture surfaces of the specimens made with different lignins were observed using SEM (Figs. 7 and 8). Neat UPE resin exhibits a relatively smooth fracture surface. There are cracks in different planes, but almost parallel to the crack-propagation direction, indicated by a white arrow (Fig. 7a and Fig. 8a).

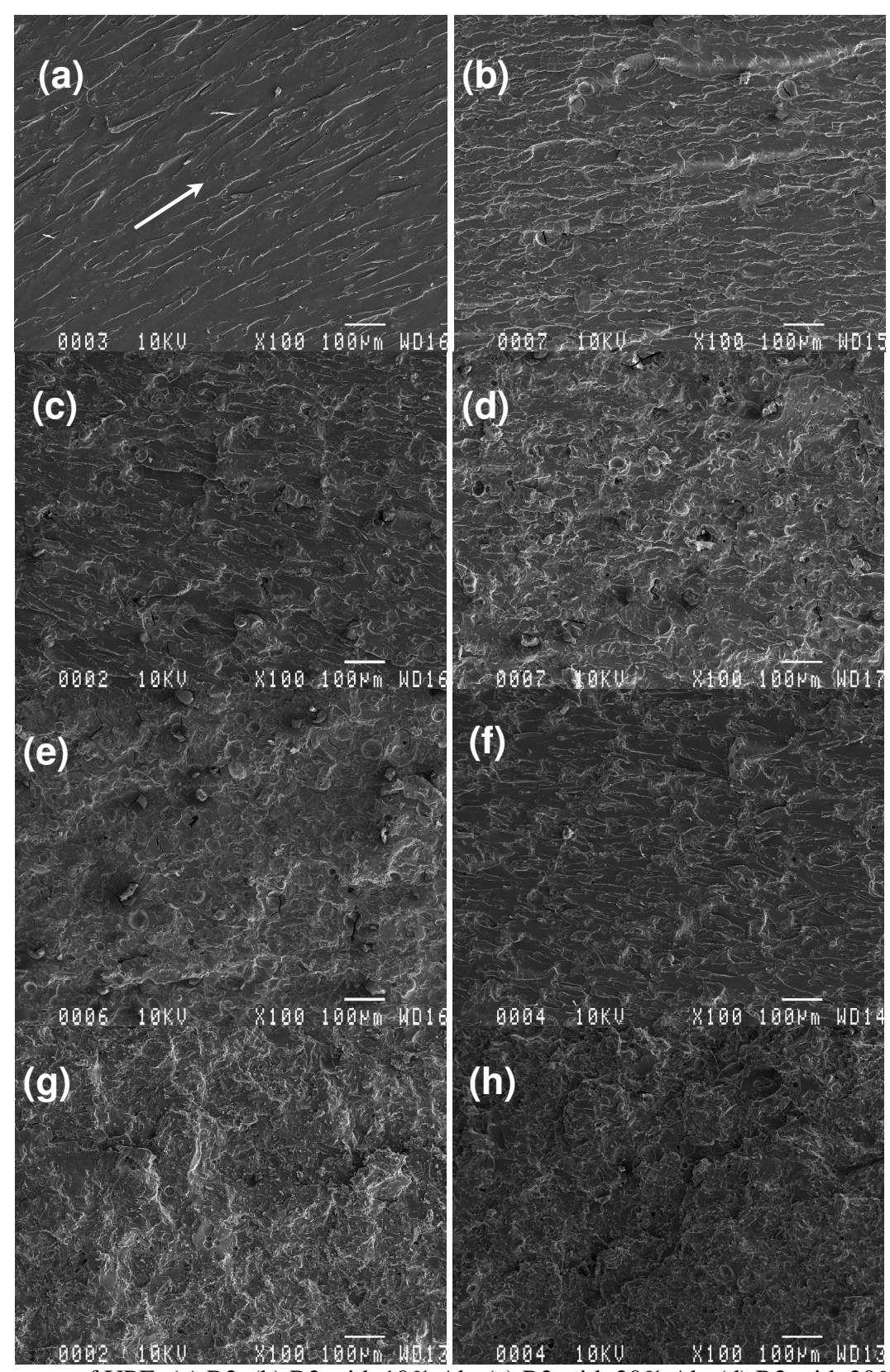

Figure 7: SEM images of UPE: (a) R2, (b) R2 with $10 \% \mathrm{Ab}$, (c) R2 with $20 \% \mathrm{Ab}$, (d) R2 with $30 \% \mathrm{Ab}$, (e) R2 with $40 \% \mathrm{Ab}$, (f) R2 with $10 \% \mathrm{AT}$, (g) R2 with $20 \% \mathrm{AT}$, and (h) R2 with $30 \%$ AT 


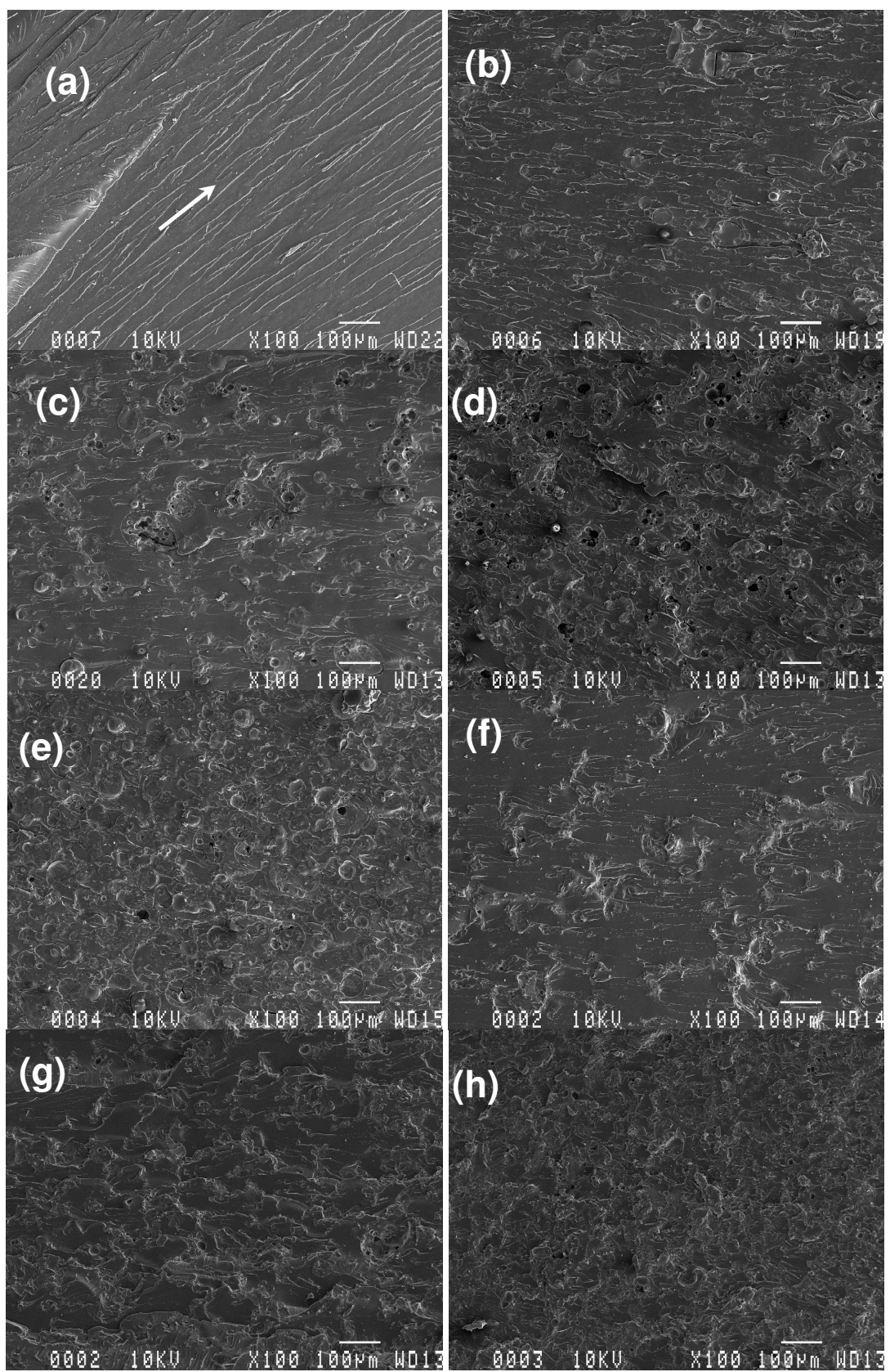

Figure 8: SEM images of UPE: (a) R9, (b) R9 with $10 \%$ Ab, (c) R9 with $20 \%$ Ab, (d) R9 with $30 \%$ Ab, (e) R9 with $40 \% \mathrm{Ab}$, (f) R9 with $10 \%$ AT, (g) R9 with $20 \%$ AT, and (h) R9 with $30 \%$ AT

This is a typical fractography feature of brittle fracture behavior, thus accounting for the low fracture toughness of the unfilled UPE. Compared to the case of neat UPE, the fracture surfaces of UPE with lignins show considerably different fractographic features. As a representative example, the failure surfaces of UPE containing two different lignins in various concentrations are shown in Figures 7 and 8 (from $b$ to h). Generally, a much rougher fracture surface is seen upon adding lignins into the UPE matrix. The increased surface roughness implies that the path of the crack tip is distorted because of the presence of lignins, making crack propagation different. More precisely, the lignin is somehow readily able to interact or interfere with the growing crack front. 
The results also show that many lignin particles are observed on the fracture surface. The presence of lignin particles or aggregates may cause perturbations along the crack front, thus altering the path of the propagating crack from the straight unperturbed growth seen in the neat resin. Consequently, the cracks are deflected by the lignin particles into the regions surrounding them.
Clearly, the crack deflection observed is expected for the increase of strength and toughness obtained by incorporating lignin into the UPE matrix. Upon fracture, the lignin particles are very likely to be the stress concentration sites; therefore, good dispersion and good interaction can help reduce the negative effect of the cleavage of lignin particles.
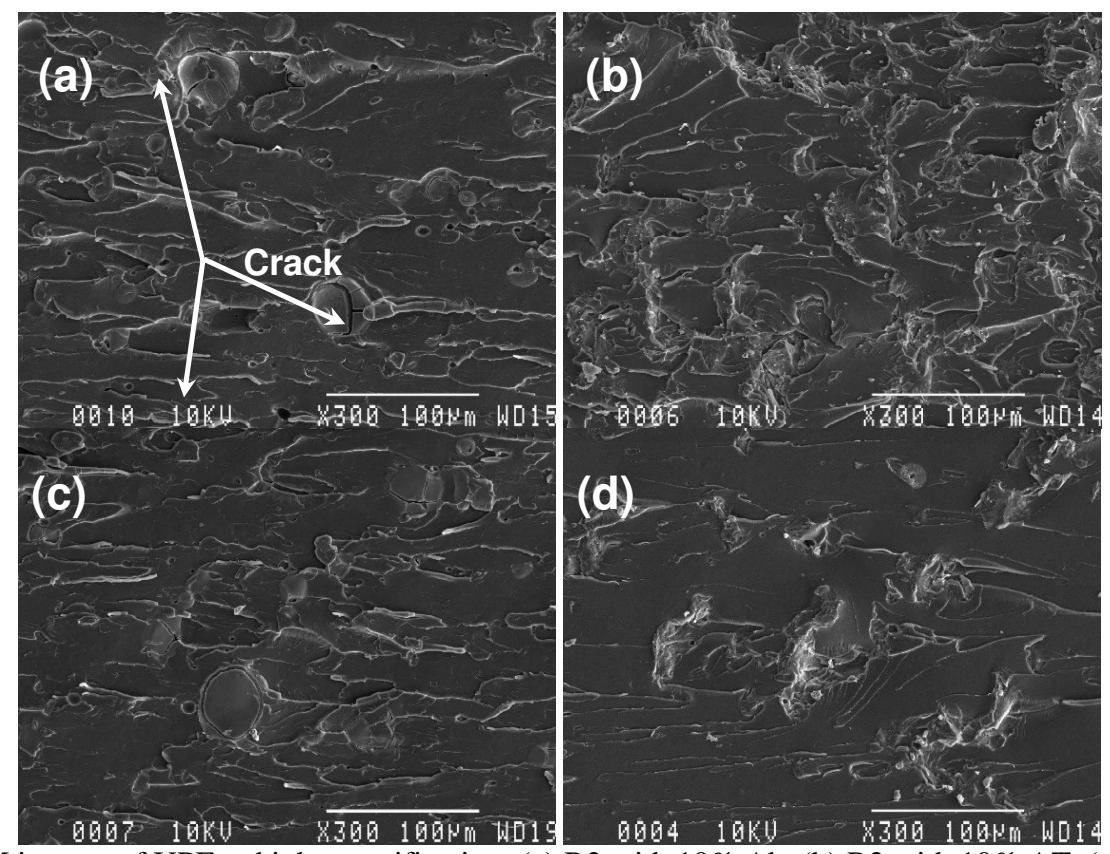

Figure 9: SEM images of UPE at high magnification: (a) R2 with 10\% Ab, (b) R2 with $10 \%$ AT, (c) R9 with 10\% $\mathrm{Ab}$, and (d) R9 with $10 \% \mathrm{AT}$

In addition, Indulin AT seems to interact better with the UPE resins than ArboSO1 under similar conditions. The crack could be observed surrounding ArboSO1 particles, as indicated in Figure 9, while this is not the case for Indulin AT. This may be related to the hydrophilicity of ArboSO1, which is not compatible with UPE resins.

\section{CONCLUSION}

Kraft and sulfite lignin samples from forestry biomass were characterized by qualitative and quantitative methods. The two lignins are quite different in terms of their chemical composition, MW, morphology, etc. The potential uses of the lignins for the preparation of UPE-lignin materials and their performance were examined.

Although the presence of lignins and their concentrations increased the viscosity of UPE, the UPE-lignins are suitable for processing. The results indicate that the lignin type and loading influenced the tensile properties of UPE. Indulin AT interacts better with UPE resins, as compared to ArboSO1. However, these factors seem not to influence the impact modulus of UPE. A much rougher fracture surface was obtained upon adding lignins into the UPE matrix.

ACKNOWLEDGEMENT: The authors would like to thank Tembec for the free ArboSO1 lignin sample.

\section{REFERENCES}

A. V. R. Prasad, K. M. M. Rao, K. M. Rao and A. V. S. S. K. S. Gupta, Indian J. Fibre Text. Res., 32, 399 (2007), http://nopr.niscair.res.in/handle/123456789/323.

2 S. Waigaonkar, B. J. C. Babu and A. Rajput, Indian J. Eng. Mater. S., 18, $31 \quad$ (2011), https://www.niscair.res.in/sciencecommunication/resea rchjournals/rejour/ijems/ijems0.asp

3 J. Martone Pt. Estevez, F. Lu, K. Ruel, M. Denny, C. Somerville et al., Curr. Biol., 19, 169 (2009), 
https://doi.org/10.1016/j.cub.2008.12.031. ISSN 09609822. PMID 19167225

4 S. E. Jr. Lebo, J. D. Gargulak and T. J. McNally, "Lignin, Kirk-Othmer Encyclopedia of Chemical Technology", John Wiley \& Sons, Inc. 2001, https://doi.org/10.1002/0471238961.12090714120914. a01.pub2

5 G. A. Smook, in "Handbook for Pulp and Paper Technologists", second edition, Vancouver, Angus Wilde Publications, 1992, pp. 5, http://vancouver.canadianorglist.com/company/anguswilde-publications-inc/

6 H. L. Hergert, in "Lignins: Occurrence, Formation, Structure and Reactions", edited by K. V. Sarkanen and C. H. Ludwig, New York, Interscience Publisher, John Wiley \& Sons, 1971, pp. 267-297, https://doi.org/10.1002/pol.1972.110100315

7 W. Thielemans and R. P. Wool, Biomacromolecules, $\quad 6, \quad 1895 \quad$ (2005), https://www.wiley.com/en-us

8 S. Gillet, M. Aguedo, L. Petitjean and A. R. C. Morais, Green Chem., 19, 4200 (2017), https://doi.org/10.1039/ C7GC01479A

9 G. A. Smook, "Handbook for Pulp and Paper Technologists", Second Edition, Angus Wilde publications, Vancouver, 1992, pp. 123-152, http://vancouver.canadianorglist.com/company/anguswilde-publications-inc/

10 R. Rinaldi, R. Jastrzebski, M. T. Clough, J. Ralph, M. Kennema et al., Angew. Chem. Int. Ed., 55, 8164 (2016), https://doi.org/10.1002/anie.201510351

11 S. Haghdan, S. Renneckar and G. D. Smith, in "Lignin Polymer Composites", edited by O. Faruk and M. Sain, Elsevier, Amsterdam, 2016, https://www.elsevier.com/books/lignin-in-polymercomposites/faruk/978-0-323-35565-0

12 S. Laurichesse and L. Avérous, Prog. Polym. Sci., 39, $1266 \quad$ (2014), https://doi.org/10.1016/j.progpolymsci.2013.11.004

13 C. Jiang, H. He, X. Yao, P. Yu, L. Zhou et al., J. Appl. Polym. Sci., 132, 1 (2015), https://doi.org/10.1002/app.42044

14 J. Datta and P. Parcheta, Iran. Polym. J., 26, 453 (2017), https://doi.org/10.1007/s13726-017-0534-0

15 J. H. Lora and W. G. Glasser, J. Polym. Environ., 10, 39

(2002), https://doi.org/10.1023/A:1021070006895

16 R. Pucciariello, V. Villani, C. Bonini, M. D'Auria and T. Vetere, Polymer, 45, 4159 (2004), https://doi.org/10.1016/j.polymer.2004.03.098

17 D. S. Argyropoulos, J. Biotechnol. Biomater., 3, 3 (2013), https://doi.org/10.4172/2155-952X.1000e123

18 M. A. S. Anwer, H. E. Naguib, A. Celzard and V. Fierro, Compos. Part B, 82, 92 (2015), https://doi.org/10.1016/j.compositesb.2015.08.028

19 H. C. Liu, C. C. Tuan, A. A. B. Davijani, P. H. Wang, H. Chang et al., Polymer, 111, 177 (2017), https://doi.org/10.1016/j.polymer.2017.01.043
20 M. Liu, A. Baum, J. Odermatt, J. Berger, L. Yu et al., Compos. Part A, 95, 377 (2017), https://doi.org/10.1016/j.compositesa.2017.01.026

21 D. Ariawan, M. S. Salim, R. M. Taib, M. Z. A. Thirmizir and Z. A. M. Ishak, Adv. Polym. Technol., 37, 1420 (2017), https://doi.org/10.1002/adv.21801

22 C. D. Tran, J. Chen, J. K. Keum and A. K. Naskar, Adv. Funct. Mater., 26, $2677 \quad$ (2016), https://doi.org/10.1002/adfm.201504990

${ }_{23}$ Y. Ikeda, T. Phakkeeree, P. Junkong, H. Yokhama, P. Phinyocheep et al., RSC Adv., 7, 5222 (2017), https://doi.org/10.1039/c6ra26359c

${ }^{24}$ L. Akim, D. Argyropoulos, L. Jouanin, J.-L. Leplé, G. Pilate et al., Holzforshung, 55, 386 (2001), https://doi.org/10.1515/HF.2001.064

25 A. Granata and D. Argyropoulos, J. Agric. Food Chem., 43, 1538 (1995). https://doi.org/10.1021/jf00054a023

26 D. Argyropoulos, J. Wood Chem. Technol., 14, 65 (1994),

https://www.tandfonline.com/doi/abs/10.1080/0277381 9408003086

27 B. Ahvazi, E. Cloutier, O. Wojciechowicz and T.D. Ngo, ACS Sustain. Chem. Eng., 4, 5090 (2016), https://doi.org/10.1021/acssuschemeng.6b00873

28 B. Ahvazi, O. Wojciechowicz, P. Xu, T.-D. Ngo and J. Hawari, BioResourcces, 12, 6629 (2017), https://doi.org/10.15376/biores.12.3.6629-6655

${ }_{29}$ G. A. Smook, in "Handbook for Pulp and Paper Technologists", edited by G. A. Smook, Angus Wilde Publications, Vancouver, 1982, pp. 66-75, http://vancouver.canadianorglist.com/company/anguswilde-publications-inc/

30 K. Koljonen, M. Österberg, M. Kleen, A. Fuhrmann and P. Stenius, Cellulose, 11, 209 (2004), https://doi.org/10.1023/B:

CELL.0000025424.90845.c3

31 G. A. Smook, in "Handbook for Pulp and Paper Technologists", edited by G. A. Smook, Angus Wilde Publications, Vancouver, 1982, pp. 58-65, http://vancouver.canadianorglist.com/company/anguswilde-publications-inc/

32 J. Gierer, J. Wood Sci. Technol., 19, 289 (1985), https://doi.org/10.1007/BF00350807

33 A. C. Neish, in "Constitution and Biosynthesis of Lignin", edited by K. Freudenberg and A. C. Neish, Springer-Verlag, Berlin, 1968, pp. 5-43, https://www.springer.com/gp/book/9783540042747

34 A. B. Wardrop, in "Lignins: Occurrence, Formation, Structure and Reactions", edited by K. V. Sarkanen and C. H. Ludwig, Interscience Publisher, John Wiley \& Sons, New York, 1971, pp. 19-41, https://doi.org/10.1002/pol.1972.110100315

35 F. E. Brauns and D. A. Brauns, "The Chemistry of Lignin”, Supplement Volume, Academic Press, New York, 1960.

36 G. G. Gross, in "The Structure, Biosynthesis and Degradation of Wood. Recent Adv. Phytochem. 11", edited by F. A. Loewus and V. C. Runeckles, Plenum 


\section{TRI-DUNG NGO et al.}

Press, New York, 1977, pp. 141-184, https://www.springer.com/gp/book/9781461588757

37 D. S. Argyropoulos, B. Hortling, K. PoppiusLevlin, Y. Sun and M. Mazur, Nord. Pulp Pap. Res. J., 10, 68 (1995), https://www.ncbi.nlm.nih.gov/nlmcatalog?term=02832631\%5BISSN\%5D

38 Y. Sun and D. S. Argyropoulos, J. Pulp Pap. Sci., 21, 185 (1995),

http://www.paptac.ca/en/publications/jppsbackissues

39 P. M. Froass, A. J. Ragauskas and J. Jiang, Pulp. J. Wood Chem. Technol., 16, 347 (1996), https://doi.org/10.1080/02773819608545820

40 H. L. Hergert, in "Lignins. Occurrence, Formation, Structure and Reactions", edited by K. V. Sarkanen and C. H. Ludwig, Wiley Interscience, New York, 1971, pp. 267-293, https://doi.org/10.1002/pol.1972.110100315

41 O. Faix, in "Methods in Lignin Chemistry", edited by S. Y. Lin and C. W. Dence, Springer-Verlag, Berlin, Germany, 1992, pp. 83-110, https://www.springer.com/gp/book/9783642740671

42 J.-S. Yeo, J.-H. Lee and S.-H. Hwang, Compos. $\begin{array}{llll}\text { Part } & \text { B, } & \text { 130, } & 167\end{array}$ https://doi.org/10.1016/j.compositesb.2017.07.084

43 A. G. Mikos and N. A. Peppas, J. Mater. Sci., 24, 1612 (1989), https://doi.org/10.1007/BF01105680 